\title{
Curative Effect Observation of Ciprofloxacin Injection in the Treatment of Acute Mastitis on Dairy Cows
}

\author{
Jie Qin \& Yanming Sun (Corresponding author) \\ College of Animal Science \& Technology \\ Shihezi University \\ Shihezi 832000, China
}

\begin{abstract}
Effects of ciprofloxacin in the treatment of acute mastitis on dairy cows was clinically investigated, penicillin-streptomycin as control. Results showed that cure rate of ciprofloxacin and penicillin-streptomycin on dairy cows was $86.0 \%$ and $72.0 \%$, respectively, and in two periods of treatment (6 days) was $72.0 \%$ and $30.0 \%$, respectively. Through statistical test, there was significance in cure rate and period of treatment between treatment group and control group, which revealed that ciprofloxacin had the characters of shorter period of treatment and better clinical efficacy.
\end{abstract}

Keywords: Ciprofloxacin, Dairy cows, Acute mastitis, Curative effect observation

\section{Introduction}

Mastitis is the most common and most costly disease of dairy cows. Among 1363 ten thousands dairy cows in China, about $40 \% \sim 70 \%$ of cows are infected with such disease. It has been estimated that mastitis costs about 3.16 hundred million yuan per annum. Acute mastitis not only results in a great economic loss, but also causes chronic mastitis subsequently, which makes galactophore tissues fibrosis and udder shrinked. Therefore, it is urgent and significant to seek a drug with high efficacy on the treatment of such mastitis. In the present paper, ciprofloxacin was applied as a drug agent to observe its curative effect.

\section{Materials and methods}

\subsection{Drugs}

Ciprofloxacin Lactate $(0.5 \mathrm{~g} / \mathrm{ml})$ was produced by Chengdu Ware Yuanheng Pharmaceutical Co.,Ltd with the batch number of 20070907; Benzylpenicillin sodium for injection (800, 000U) was produced by Jiangsu Nannong Gaoke Animal Pharmaceutical Co., Ltd with the batch number of 20070107; Streptomycin sulfate for injection (1000, 000U) was also produced by Jiangsu Nannong Gaoke Animal Pharmaceutical Co., Ltd with the batch number of 20070526. All these drugs above were purchased from Shihezi veterinary station without further purification.

\subsection{Experimental methods}

\subsubsection{Animals}

All dairy cows in the treatment were diagnosed as having acute mastitis in several cattle farms of Kuidun in 2008. Symptoms of cows with acute clinical mastitis were described as follows: Inflammation is characterized by gross abnormalities in the udder (swelling, heat, redness, pain) and change in composition and appearance of milk. Abnormalities in milk may include flakes, clots or a watery appearance. The flakes in the milk are congealed leukocytes, secretory cells and protein.

\subsubsection{Allocation of treatment}

100 cows infected during experiment were randomly divided into two groups, ciprofloxacin group (treatment group) and penicillin-streptomycin (control group), 50 per group. For ciprofloxacin group, infusion through udder at a dose of $20 \mathrm{ml}$, once a day, 3 days a period of treatment; For penicillin-streptomycin group, infusion through udder at a dose of $800,000 \mathrm{U}$ penicillin and 1000,000U streptomycin together (diluted by normal saline to $50 \mathrm{ml}$ ), twice a day, 3 days a period of treatment.

\subsubsection{Criterion of curative effect}

According to the criterion of Zhou Xuzheng (2007, PP. 44-47), briefed as follows:

Cure: clinical symptoms vanished and the properties, color and yield of milk all recovered to normal.

Validity: clinical symptoms basically vanished with increased milk yield and property and there were still few clots in 
the milk.

Invalidation: Clinical symptoms and properties of milk failed to develop improvement apparently, or even worse.

\subsubsection{Data analysis}

Data were analyzed statistically by the software SPSS 3.0; Valued of $\mathrm{P}<0.05$ were regarded as significant.

\section{Results}

\subsection{Curative effect}

Cure rate of ciprofloxacin and penicillin-streptomycin in the treatment of acute mastitis on dairy cows was $86.0 \%$ and $72.0 \%$, respectively. Through statistical test, it showed that ciprofloxacin group got best efficacy with great significance. The detail results were listed in Table 1.

\subsection{Period of treatment}

Three days applied as a period treatment, according to specific curative effect, 1 to 3 periods of treatment were performed. Periods of treatment showed great significance between two groups through statistical test, which were listed in Table 2.

\section{Discussions}

Amongst effects of ciprofloxacin and penicillin-streptomycin in the treatment of acute mastitis on dairy cows, ciprofloxacin showed its best efficacy and penicillin-streptomycin followed. Effect of ciprofloxacin in the present paper basically conformed to the investigation of Zhang Jinhe (2007, PP.36-38).

Research showed that fluoroquinolone consumption was feasible to inhibit DNA synthesis of bacteria and have no crossing drug-resistance. Compared to other antibiotics, it was not susceptible to result in drug-resistance. Ciprofloxacin could accumulate in phagocytic cell, which achieved possibility to kill mastitis pathogens.

As far as period in the treatment of acute mastitis is concerned, the period of ciprofloxacin was shortest with great significance, compared to penicillin-streptomycin. In the present experiment, curative rate of ciprofloxacin in two periods of treatment achieved $72.0 \%$ with great significance, compared to $30 \%$ of penicillin-streptomycin. Cure rate of ciprofloxacin was $86.0 \%$, and total validity rate was $94.0 \%$. Ciprofloxacin had shorter period of treatment as well. Taken together, ciprofloxacin could be used as a desirable drug agent in the treatment of acute mastitis and developed to further widespread application.

\section{References}

Zhang, Jinhe, Han, Min, \& Zhao, Suwei. (2007). Clinical Study of Ciprofloxacin Lactate Injection in the Treatment Acute Mastitis on Dairy Cow. China Dairy Cattle, 8:36-38.

Zhou, Xuzheng. (2007). The Study of "Ruyuankang" on Treatment Trial of Dairy Cow Mastitis. Progress in Veterinary Medicine, 28(2):44-47.

Table 1. Curative effect of two groups on acute mastitis

\begin{tabular}{|c|c|c|c|c|}
\hline \multirow[t]{2}{*}{ Curative Effect } & \multicolumn{2}{|c|}{$\begin{array}{l}\text { Penicillin-streptomycin } \\
\text { Group }(50)\end{array}$} & \multicolumn{2}{|c|}{ Ciprofloxacin Group (50) } \\
\hline & Case Load & $(\%)$ & Case Load & $(\%)$ \\
\hline Cure & 36 & $72.0^{\mathrm{a}}$ & 43 & $86.0^{\mathrm{b}}$ \\
\hline Validity & 3 & $6.0^{\mathrm{a}}$ & 4 & $8.0^{\mathrm{a}}$ \\
\hline Invalidation & 11 & $22.0^{\mathrm{a}}$ & 3 & $6.0^{\mathrm{b}}$ \\
\hline Total validity & 39 & $78.0 \%{ }^{\mathrm{a}}$ & 47 & $94.0 \%^{\mathrm{b}}$ \\
\hline
\end{tabular}


Table 2. Period of treatment of two groups against acute mastitis

\begin{tabular}{|c|c|c|c|c|}
\hline \multirow{2}{*}{$\begin{array}{l}\text { Period of } \\
\text { treatment }\end{array}$} & \multicolumn{2}{|c|}{ Penicillin-streptomycin Group $(50)$} & \multicolumn{2}{|c|}{ Ciprofloxacin Group (50) } \\
\hline & \multicolumn{2}{|c|}{ Cure Case $(\%)$} & \multicolumn{2}{|c|}{ Cure Case $(\%)$} \\
\hline 1 period & & $10.0^{\mathrm{a}}$ & 16 & $32.0^{\mathrm{b}}$ \\
\hline 2 period & 10 & $20.0^{\mathrm{a}}$ & 20 & $40.0^{\mathrm{b}}$ \\
\hline 3 period & 21 & $42.0^{\mathrm{a}}$ & 7 & $14.0^{\mathrm{b}}$ \\
\hline
\end{tabular}

